\title{
Metabolomics of exhaled breath condensate: a means for phenotyping respiratory diseases?
}

\author{
"We believe that metabolomic profiling of EBC could have a \\ widespread application in phenotyping respiratory disorders."
}

First draft submitted: 28 February 2017; Accepted for publication: 18 March 2017; Published online: 15 June 2017

\section{Keywords: asthma $\bullet$ COPD $\bullet$ inflammation $\bullet$ lung $\bullet$ metabolomics $\bullet$ respiratory}

Respiratory diseases are widely spread worldwide [1]. Among them, bronchial asthma and chronic obstructive pulmonary disease (COPD) affect several million people [1], with COPD being one of the major causes of death. Respiratory diseases are complex and heterogeneous pathologies, and bring about an increase in morbidity, mortality and healthcare expenses. The complexity of these diseases is linked to multiple mechanisms that are not present in all patients at any given time point, or in the same patient at different time points [2], and these features underline the presence of specific phenotypes. A phenotype is operationally defined as "a cluster of features that leads to the separation of a specific group at a given time" [3]. Therefore, what is required is the identification of specific phenotypes, which recognize groups of patients with similar characteristics, prognosis or treatment needs, paving the path to personalized respiratory medicine [4].

Phenotypes can be studied by looking for specific biomarkers. However, complex and heterogeneous disorders, such as asthma or COPD, are not characterized by a single biomarker, and even diseases presenting a single genetic abnormality like cystic fibrosis (CF), display multiple biomarkers. In general, a panel of biomarkers is required for an accurate description of the molecular aspects of a disease.

\section{Metabolomics}

In the last few years, 'omics' sciences have improved disease phenotyping by linking the molecular mechanisms to the clinical field [5]. In particular, metabolomics has become a leading tool in defining disease phenotype because it can read the impairment induced by the pathogenic mechanisms, and it is able to correlate the dysregulation and the associated phenotype. Since the metabolome is a downstream system of the proteome and transcriptome, it has been shown to be amplified [6], and therefore it represents a more sensitive level of organization than the proteome or transcriptome for understanding a complex biological outcome like respiratory diseases.

Metabolomics makes no assumptions on the underlying mechanisms of disease, and it is concerned with the identification and quantification of small molecules $(<1500$ Da) in biological specimens, including body fluids. It provides a 'top-down' approach that gives an integrated view of the system as opposed to the 'bottom-up' approach where the effects of an individual gene, or groups of genes, are investigated [5]. Furthermore, metabolomics investigates a relatively small number of human metabolites (ca. 7000), as compared with the estimated numbers of genes (ca. 25,000), transcripts (ca. 100,000) and proteins (ca. 1,000,000).

\section{Application of metabolomics to exhaled breath condensate}

Metabolomics is a useful tool to investigate airways diseases, as the respiratory tract offers a natural matrix (exhaled breath), which is easily collected as exhaled breath condensate (EBC), and contains several biomarkers of

\section{Biomarkers \\ in Medicine}
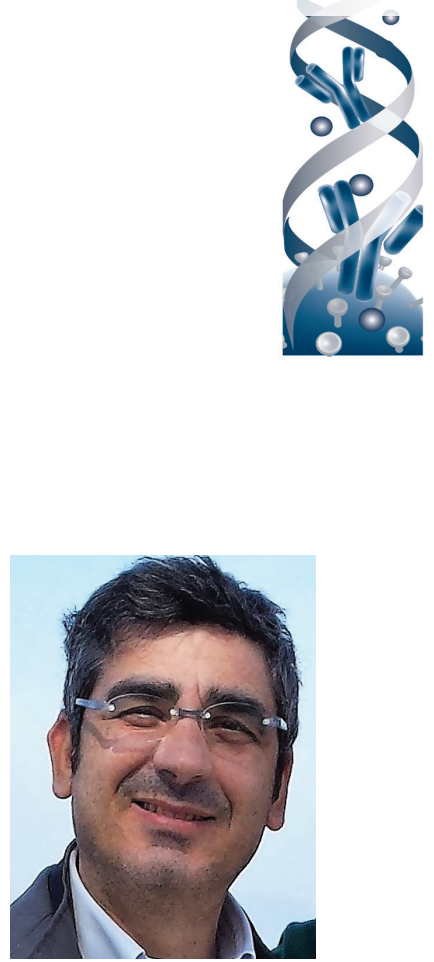

Mauro Maniscalco Author for correspondence: Pulmonary Rehabilitation Unit, ICS Maugeri SPA, IRCCS, 82037 Telese Terme, Benevento, Italy Tel.: + 390824909350 Fax: + 390824909614 mauro.maniscalco@icsmaugeri.it

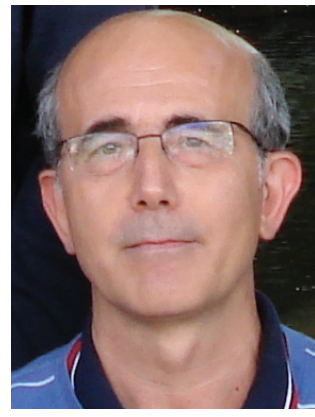

Andrea Motta ${ }^{2}$ Institute of Biomolecular Chemistry, National Research Council, 80078 Pozzuoli, Naples, Italy

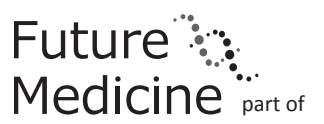


airway inflammation and oxidative stress [7]. EBC is collected by cooling the exhaled breath, obtaining a liquid that contains evaporated and condensed particles derived directly from the airway lining fluid [8]. It is assumed that airway surface liquid becomes aerosolized during turbulent airflow; therefore, the condensate reflects the composition of airway surface liquid, although large molecules may not aerosolize. EBC is mainly formed by water vapor ( $>99 \%)$, but it also contains leukotrienes, 8-isoprostane, prostaglandins, hydrogen peroxide, nitric oxide-derived products, hydrogen ions [8], as well as amino acids, short-chain fatty acids, etc.

\section{"Recently, our group characterized the respiratory metabotype of obese asthmatic patients, and compared it to that of lean asthmatic patients."}

Several different technological platforms are available to analyze the metabolic profile, but MS and NMR spectroscopy are the most widely used [9]. When working with biological fluids, NMR often does not require chemical manipulation of the sample, whereas MS usually necessitates derivatization of metabolites to produce ionic species that are more readily separated by $\mathrm{m} / \mathrm{z}$ ratio. NMR spectroscopy also remains popular for untargeted metabolic profiling because of its simplicity, broad metabolite detection capabilities, robustness and repeatability. MS presents a strong advantage in sensitivity compared with NMR, and by using different combinations of chromatographic methods and mass/charge separation technologies MS is able to detect a broader array of metabolites. NMR and MS are often used in combination as they provide complementary information. The datasets obtained by both techniques are interpreted using multivariate analysis methods such as principal component analysis and partial least squares projection to latent structures.

\section{Phenotyping EBC}

Carraro et al. [10] first applied NMR-based metabolomics to EBC of asthmatic children, who were distinguished from healthy controls by the variation of the acetate signal. The authors speculated that such enhancement was due to an increased acetylation of proinflammatory proteins in the extracellular space of the airway environment. Application to EBC samples from adults has also been reported [11]. By using a similar approach, de Laurentiis et al. evaluated the possibility to separate COPD patients from healthy subjects, detecting qualitative and quantitative differences in five NMR signals in EBC samples. Lately, Motta et al. compared mild asthmatic adult patients with healthy subjects, finding a clear separation between the groups [12].
The first use of EBC for phenotyping a respiratory disease was done by de Laurentiis et al. [13], who studied patients affected by smoking-related diseases with similar pathogenesis. It was found that patients exposed to the same risk factor, namely, smoking habit in COPD smokers and subjects with established pulmonary Langerhans cell histiocytosis (PLCH), could be easily separated detecting an inverse behavior of 2-propanol and isobutyrate in COPD with respect to $\mathrm{PLCH}$ (high/low in COPD, low/high in PLCH) for each metabolic phenotype (metabotype).

Bertini et al. investigated whether EBC from COPD patients features distinct metabolic fingerprints (phenotypes). They reported that COPD samples were divided in three clusters, one of which had the highest prevalence of radiologic emphysema [14], proving the power of metabolomics in identifying different metabotypes within a specific pathology.

EBC was also studied to characterize asthma phenotypes in children. In 2013, Carraro et al. [15] discriminated different asthma phenotypes, with a particular focus on severe asthma in children. They found that compounds related to retinoic acid, adenosine and vitamin $\mathrm{D}$ were relevant for the separation of different groups. In particular, a robust partial least square analysis allowed discrimination between severe and nonsevere asthma.

Ibrahim and colleagues [16] determined and validated discriminatory metabolomic profiles in adult asthmatic patients. In this study, asthma phenotypes based on sputum inflammatory cells, asthma control and inhaled corticosteroid use were investigated. The authors could distinguish asthmatics based on inhaled corticosteroid use and sputum neutrophilia, but they were not able to differentiate the asthma phenotypes of sputum eosinophilia and asthma controls.

Recently, our group characterized the respiratory metabotype of obese asthmatic patients [17], and compared it to that of lean asthmatic patients [18]. We found that patients affected by asthma associated with obesity express a respiratory metabotype that is fully different, not summatory, with respect to those separately characterizing patients with either asthma or obesity alone. The metabolic signature we described for obese asthmatic patients can be validly considered a specific manifestation of an obese-asthmatic respiratory phenotype, based on both conceptual and experimental evidence [18].

EBC metabolomics is also capable to phenotype diseases characterized by airway-ciliated epithelium genetic abnormalities such as CF. Montuschi et al. [19] evaluated the possibility to discriminate between patients with stable and unstable CF by NMR-based metabolomics. In their controlled and validated study, 
the authors found that seven metabolites were able to distinguish stable versus exacerbation samples, with possibility to use only four metabolites (ethanol, acetate, 2-propanol and methanol) without significant loss in sensitivity and specificity. The CF respiratory phenotype was compared with that of primary ciliary dyskinesia. In a cross-sectional study, Montuschi et al. [20] separated primary ciliary dyskinesia and CF identifying selective metabolites (ethanol, methanol and short chain fatty acids) accounting for their differing prognoses.

\section{Conclusion}

We believe that metabolomic profiling of EBC could have a widespread application in phenotyping respiratory disorders. Furthermore, from 'profiling' EBC,

\section{References}

1 Bousquet J, Dahl R, Khaltaev N. Global alliance against chronic respiratory diseases. Allergy 62(3), 216-223 (2007).

2 Agusti A. The path to personalised medicine in COPD. Thorax 69(9), 857-864 (2014).

3 Bush A, Fleming L. Phenotypes of refractory/severe asthma. Paediatr. Respir. Rev. 12(3), 177-181 (2011).

4 Woodruff PG, Agusti A, Roche N, Singh D, Martinez FJ. Current concepts in targeting chronic obstructive pulmonary disease pharmacotherapy: making progress towards personalised management. Lancet 385(9979), 1789-1798 (2015).

5 Lindon JC, Nicholson JK. The emergent role of metabolic phenotyping in dynamic patient stratification. Expert Opin. Drug Metab. Toxicol. 10(7), 915-919 (2014).

6 Urbanczyk-Wochniak E, Luedemann A, Kopka J et al. Parallel analysis of transcript and metabolic profiles: a new approach in systems biology. Embo. Rep. 4(10), 989-993 (2003).

7 Sofia M, Maniscalco M, De Laurentiis G, Paris D, Melck D, Motta A. Exploring airway diseases by NMR-based metabonomics: a review of application to exhaled breath condensate. J. Biomed. Biotechnol. 2011, 403260 (2011).

8 Horvath I, Hunt J, Barnes PJ et al. Exhaled breath condensate: methodological recommendations and unresolved questions. Eur. Respir. J. 26(3), 523-548 (2005).

9 Lindon JC, Nicholson JK. Spectroscopic and statistical techniques for information recovery in metabonomics and metabolomics. Annu. Rev. Anal. Chem. (Palo Alto Calif). 1, 45-69 (2008).

10 Carraro S, Rezzi S, Reniero F et al. Metabolomics applied to exhaled breath condensate in childhood asthma. Am. J. Respir. Crit. Care Med. 175(10), 986-990 (2007).

11 De Laurentiis G, Paris D, Melck D et al. Metabonomic analysis of exhaled breath condensate in adults by nuclear it is possible to map dysregulated metabolic pathways involved in the pathophysiological mechanisms of diseases. This will allow identification of novel mechanisms and therapeutical targets for a personalized respiratory medicine through more 'tailored' approaches.

\section{Financial \& competing interests disclosure}

The authors have no relevant affiliations or financial involvement with any organization or entity with a financial interest in or financial conflict with the subject matter or materials discussed in the manuscript. This includes employment, consultancies, honoraria, stock ownership or options, expert testimony, grants or patents received or pending, or royalties.

No writing assistance was utilized in the production of this manuscript.

magnetic resonance spectroscopy. Eur. Respir. J. 32(5), 1175-1183 (2008).

12 Motta A, Paris D, D’Amato M et al. NMR metabolomic analysis of exhaled breath condensate of asthmatic patients at two different temperatures. J. Proteome Res. 13(12), 6107-6120 (2014).

13 De Laurentiis G, Paris D, Melck D et al. Separating smoking-related diseases using NMR-based metabolomics of exhaled breath condensate. J. Proteome Res. 12(3), 1502-1511 (2013).

14 Bertini I, Luchinat C, Miniati M, Monti S, Tenori L. Phenotyping COPD by H-1 NMR metabolomics of exhaled breath condensate. Metabolomics 10(2), 302-311 (2014).

15 Carraro S, Giordano G, Reniero F et al. Asthma severity in childhood and metabolomic profiling of breath condensate. Allergy 68(1), 110-117 (2013).

16 Ibrahim B, Marsden P, Smith JA, Custovic A, Nilsson M, Fowler SJ. Breath metabolomic profiling by nuclear magnetic resonance spectroscopy in asthma. Allergy 68(8), 1050-1056 (2013).

17 Paris D, Maniscalco M, Melck D et al. Inflammatory metabolites in exhaled breath condensate characterize the obese respiratory phenotype. Metabolomics 11(6), 1934-1939 (2015).

18 Maniscalco M, Paris D, Melck DJ et al. Coexistence of obesity and asthma determines a distinct respiratory metabolic phenotype. J. Allergy. Clin. Immunol. 32(2), 472-486 (2016).

19 Montuschi P, Paris D, Melck D et al. NMR spectroscopy metabolomic profiling of exhaled breath condensate in patients with stable and unstable cystic fibrosis. Thorax 67(3), 222-228 (2012).

20 Montuschi P, Paris D, Montella S et al. Nuclear magnetic resonance-based metabolomics discriminates primary ciliary dyskinesia from cystic fibrosis. Am. J. Respir. Crit. Care Med. 190(2), 229-233 (2014). 